\title{
Rapidly Gathering linformation in Wireless Sensor Networks using Demand Assigned Multiple Access (DAMA) with Multiple Access Collision Avoidance (MACA)
}

\author{
G. Vennila", D. Arivazhagan and N. Manickasankari
}

Department of Information Technology, AMET University, Chennai, India; g.vennila265@gmail.com, arivazhagand@hotmail.com, manickasankari@gmail.com

\begin{abstract}
The most essential application in Wireless Sensor Network (WSN) is Data gathering. The converge cast is method for collecting the data from several sensor nodes to sink node. During data gathering, there is the possibility of occurring collision, retransmission etc. One of the major problems in data gathering is collision. To deal with this particular data collection problem, several techniques are proposed for efficient and fast data collection in Wireless Sensor Network. This paper uses Demand Assigned Multiple Access (DAMA) to maintain scheduling protocol for data gathering in the depiction of tree-based structure in which the packets are collected based on the user demand within the communication range and Multiple Access with Collision Avoidance (MACA) provides fast data transmission due to avoiding the collisions which provides an efficient result. Thus the throughput has been increased and delay reduced for data collection in wireless sensor network.
\end{abstract}

Keywords: Data Collection, DAMA, MACA, TDMA, Wireless Sensor Network

\section{Introduction}

The converge cast is one of the data gathering applications in Wireless Sensor Network (WSN). It collects the data from sensor nodes to base station in a tree based structure ${ }^{1}$. Collecting information at the node level such as computing the sum or the average of sensor node reduces the need for communication instead of transmitting the packets of each node separately; a node collects the incoming packets within communication range and communicates the collected information to the next node in the collection path ${ }^{2}$. Energy efficiency and time efficiency are two major considerations for sensor data collection in wireless sensor networks ${ }^{3}$. The DAMA (Demand Assigned Multiple Access) is a reservation scheme and always combines with TDM (Time Division Multiplexing). The main objective of this paper is to provide rapid collection of data to improve the performance of WSN. The paper implements the idea of Multiple Access with Collision Avoidance to remove the collisions and retransmission.

\section{Problem Statement}

Most of the previous methods encompass the problem with base station. The problem in Data gathering is that the sensor host communicated with other host is hidden for base station. All of these methods with base station use TDMA. So, the difficulty of hidden host is unidentified. This paper defines an algorithms based on DAMA and MACA in terms of Throughput and Delay.

\section{Data Collection in WSN}

Data collection is one of the fundamental tasks in Wireless Sensor Network. While collecting the data from sensor nodes to base station (sink) in which the flow of data from

*Author for correspondence 
many sensor nodes to one sink node ${ }^{4}$. So, this is called as many - to - one communication as shown in Figure 1.

The data convergecast (data collection) has two types 1) Aggregated data and 2) Raw data collection. In the aggregated data collection, the packets are sent from sensor to the local sink or relay node and then those data's are get transmitted into the sink node ${ }^{5}$. In the raw data collection method, the packets are honestly transferred to the sink node without transferring to the aggregated node or relay node. The data convergecast is also called as data gathering ${ }^{6}$.

\section{Proposed Work}

This paper gives better result to avoid collision and retransmission by using DAMA and MACA. The proposed algorithm has two parts 1) Assigning Timeslot 2) Data Transmission. It uses the concept of both DAMA and MACA.
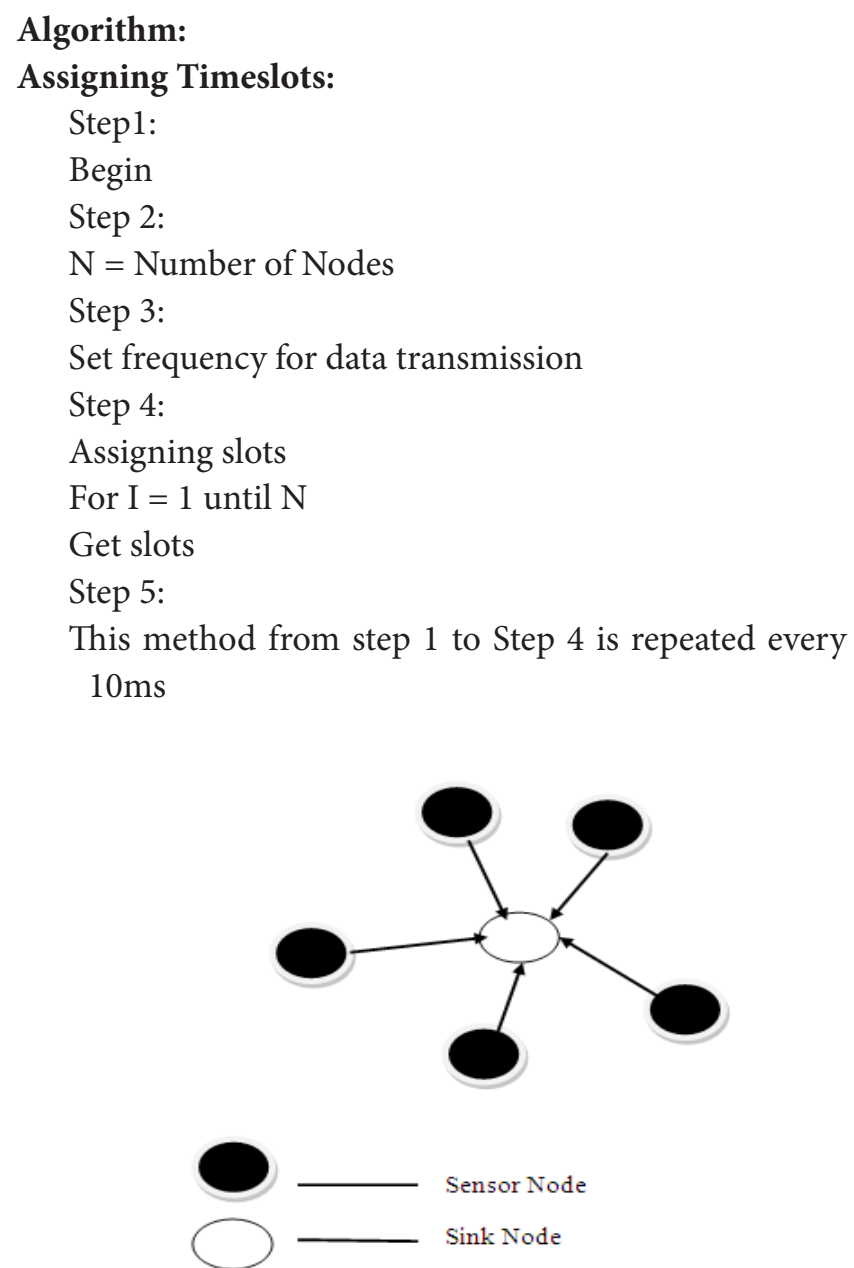

Figure 1. Many - to - one communication.

\section{Data Transmission:}

Step 6:

Begin Transmission

Step 7:

Initially Bs = Idle

Step 8:

Bs issues RTS (Request to Send)

Step 9:

Wait

Step 10:

Send CTS (Clear to Send)

Step 11:

If the Bs gets CTS, it sends packets by using the followings,

For $\mathrm{i}=1$ until $\mathrm{N}$

Send packets

Otherwise Bs again issues RTS after timeout

Step 12:

After successful transmission, the Bs waits for ACK to return into Idle.

Step 13:

When the sink node wants to collect the data from Sensor node based on timeslots, the above procedure is repeated from Step 5 to Step 12.

In Assigning timeslots, the slots are assigned by using the method of DAMA that combine with Time Division Multiplexing. In Data Transmission, the packets are transmit from sensor node to base station is done via Multiple Access with Collision Avoidance.

\subsection{Simulation Results}

The simulation was carried out using MATLAB. The MATLAB provides a simple platform for simulation. The number of sensor nodes from 10 to 50 is distributed equally within the area $1000 \mathrm{X} 1000$. The simulation is run for 650 seconds therefore the algorithm has enough time to collects the data from the sensor node to sink node. The following performance metrics are considered to evaluate the performance of the network in terms of Throughput and Delay as given below:

\section{Throughput:}

The process of taking the time to successful transmission through the communication path is called as throughput.

Delay:

The time taking to transfer the packets from source to destination is called as delay. 
The performance of network throughput is shown in Figure 2.

The performance of network delay is shown in Figure 3.

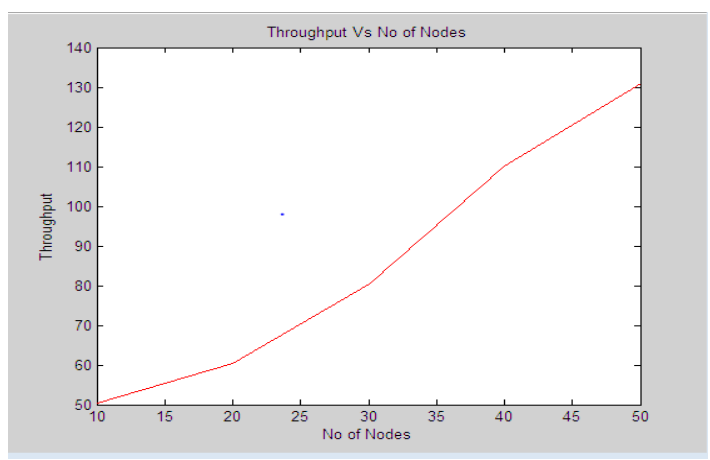

Figure 2. Throughput Vs number of nodes.



Figure 3. Delay Vs number of nodes.

\section{Conclusion}

This paper proposed two algorithms based on Demand Assigned Multiple Access and Multiple Access with Collision Avoidance for rapid data collection in WSN. The Demand Assigned Multiple Access that collaborates through Time division Multiplexing used to assign time slots for well-organized data communication. The Multiple Access with Collision Avoidance used to avoid collisions during data communication. These two techniques provides high throughput due to eliminating the collisions and minimizing the delay.

\section{References}

1. Pavitha N. Fast and efficient data collection in wireless sensor networks: a survey. IJR. 2014 Aug; 1(7):126-30. ISSN 2348-6848.

2. Kulik L, Tanin E, Umer M. Efficient data collection and selective queries in sensor networks. Springer: Verlag Berlin Heidelberg; 2008.

3. Yogeswaril R, Subathra V. A survey on efficient data collection in wireless sensor network. IJIRCCE. 2013 Nov; 1(9):2181-4.

4. Dubey S, Agrawal C, Jain A. The hybrid algorithm for data collection over a tree topology in WSN. Comput Eng Intell Syst. 2014; 5(4):65-79.

5. Jayachandran J, Ramalakshmi R. Fast data collection with reduced interference and increased life time in wireless sensor net. IJREAT. 2013 Apr-May; 1(2):1-5. 